(1) Levis - $\frac{1}{2}+2$.

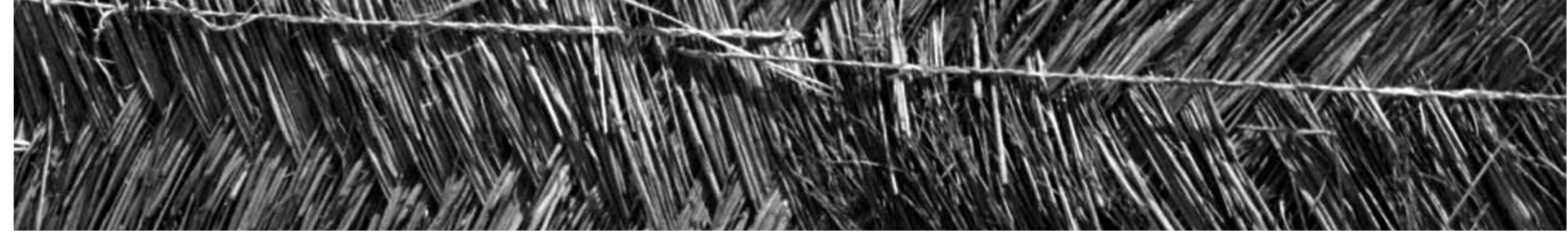




\section{Ground-breaking: Scientific and Sonic Perceptions of Environmental Change in the African Sahel}

\author{
W. Paul Adderley \\ and Michael Young
}

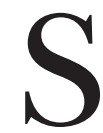

ocieties are often required to react to environmental events that arise through either anthropogenic or natural processes. The extremity of such events can be measured in terms of their immediacy and intensity and by how readily they can be comprehended when set against established understandings. For example, our present-day debate on future climatic change is driven by scientific assertion, reinforced by evidence gathered from both instrument and proxy measurements, while the varying societal responses are predicated by everyday cultural experiences. Climate-change denial is one such example of a cultural response. In contrast, places considered to offer experiences at the boundaries or outside the everyday, for example, hot and cold deserts, provide a different conception of extreme, in which change and the rates of change typically lack context and validation within everyday norms. Consequently, it is within such surroundings that the greatest tension occurs between the perceptions of place, rates of change and extremity-perceptions that can be interrogated through both positivistic scientific methods and "nonrational" art-practice. Whether or not these customary characterizations are legitimate, the obvious epistemological differences between scientific and artistic enquiry can both illuminate and problematize our understanding. In this paper, we describe and reflect upon a real-time generative installation we created that attempts to explore and test these differences: Ground-breaking: Extreme Landscapes in Grains and Pixels [1] (excerpts are available on-line [2]).

This work offers context and potential validation regarding change and the rate of change in an environment, evidenced through scientific analysis of landscapes and soils. This evidence is translated to create an audiovisual installation through a process of critical evaluation. The installation seeks to convey the cultural imprints left by societal responses to change

W. Paul Adderley (scientist, educator), School of Biological and Environmental Sciences, University of Stirling, Stirling FK9 4LA, Scotland, U.K. E-mail: <w.p.adderley@stir.ac.uk>.

Michael Young (composer, educator), Music Department, Goldsmiths, University of London, New Cross, London SE14 6NW, U.K. E-mail: <m.young@gold.ac.uk>.

Based on a paper presented at the international conference MutaMorphosis: Challenging Arts and Sciences <www.mutamorphosis.org >, 8-10 November 2007, Prague, Czech Republic, organized by CIANT (International Centre for Art and New Technologies) and co-organized by Leonardo/ISAST, as part of the ENTER 3 festival.

See $<w w w . m i t p r e s s j o u r n a l s . o r g / t o c / l e o n / 42 / 5>$ for supplemental files related to this article.

Article Frontispiece. House building at Zilum Kura, North East Nigeria. (Photo @ Paul Adderley) experienced in a geographically and socially marginalized area, the African Sahel [3]. By considering a landscape that is both extreme and has long-standing cultural activity, a narrative is developed. To borrow Barthes's terminology [4], the data from scientific analysis provide functions to the narrative; they are indices to the landscape and to human conditions. These data also connote actions that may be anthropogenic or environmental (such as changes in land-management, flooding and desertification). A narrative emerges from the exploration of these data, in which a sequence of actions is deduced from functional descriptions of physical objects, which in turn are offered for evaluation and exploration in sonic and visual forms.

\section{CONTEXT AND INVESTIGATION}

In both extreme and non-extreme environments, the fertility of soil is fundamental to the long-term sustainability of human societies. The local management of soils is paramount in agrarian-based societies, whereas nomadic pastoralist societies are reliant at more regional scales. Soils are, therefore, both an essential source of, and sink for, materials used to sustain basic human existence, providing nutrients for plant growth and receiving material inputs in the form of wastes or fertilizers. Unless lost by erosion, soils subjected to such inputs will retain an imprint of past cultural activities. This imprint, commonly microscopic, provides the opportunity to examine how past societies managed their surrounding landscapes. Soils therefore can act as a record of past cultural activities; the examination of such soils-cultural soils-is a major element in the Groundbreaking installation.

The soil materials used as a basis for the installation were sampled from the Sahel region of West Africa from a village called Tiwa. The village is located in the lacustrine plain of Lake Chad in Northern Nigeria [5] and is representative of many thousands of villages in the region [6]. The lacustrine plain has experienced extremes of flooding and drought throughout history that may have displaced human populations. The village lies on a raised mound-the surrounding plain may flood with seasonal rains-with dwellings constructed from mud-brick and thatch (Fig. 1 and Article Fron- 


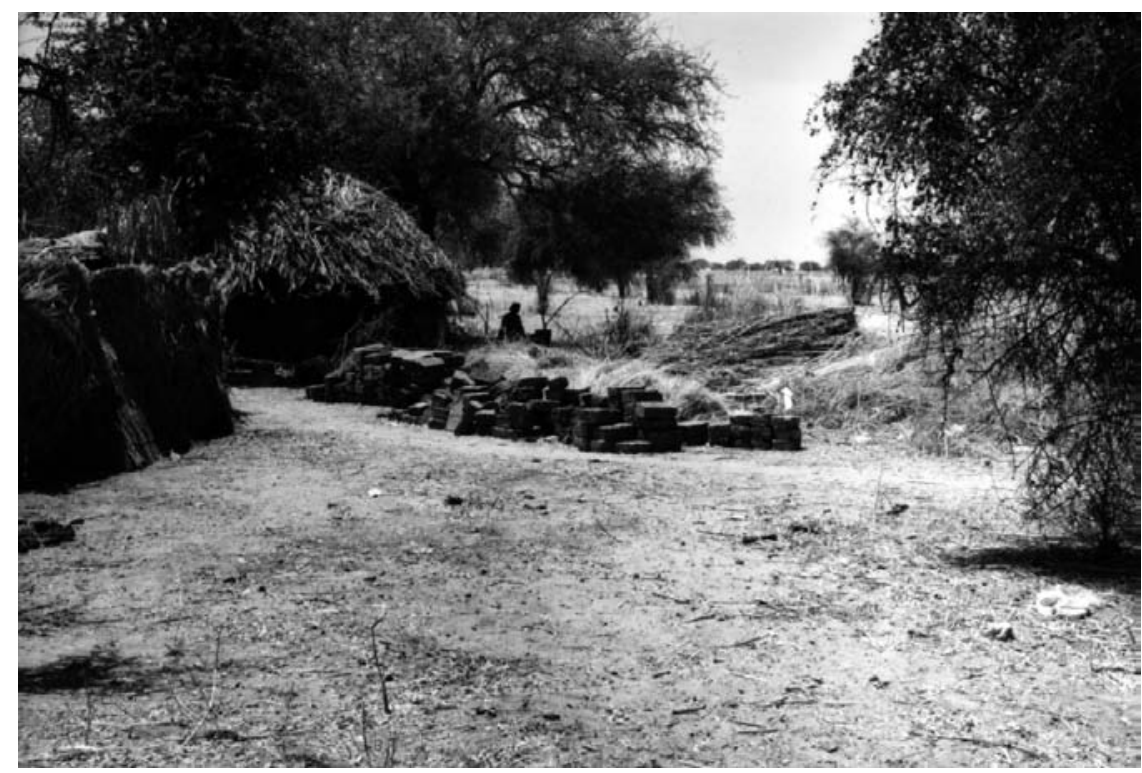

Fig. 1. Modern-day production of mud-brick used for building houses and animal enclosures in a village south of Lake Chad in northeastern Nigeria. (Photo () Paul Adderley) These materials appear have been used in this region through the period of settlement of the past four millennia [5]. Forensic examination of soils surrounding villages reveals a cultural imprint that includes fragments of such materials.

tispiece). Soil materials were sampled by Paul Adderley from a field on the village fringe. This area is typically cared for by women and small children and is subject to intense land management; it receives domestic and animal wastes as well as materials washed in by rain. A pit, $2 \mathrm{~m}$ deep, was dug to expose a profile for sampling. The soil materials were sampled as blocks using open-faced containers called Kubiëna tins. These allow the spatial organization of the soil material to be maintained throughout the handling of the material, from sampling, through subsequent laboratory processing, to examination by microscope (Fig. 2). With the deeper, that is, older, cultural soils at depths undisturbed by modern-day ac- tivities, an intact imprint of past human activities may be retained. The oldest soil materials were subsequently found to be c. 10,000 years old. The materials sampled therefore span this 10,000-year period, including the onset of human settlement in the Lake Chad plain c. 4,000 years ago [7].

We treated the soil materials to produce glass-mounted thin-section samples for examination by transmitted and reflected light using an optical microscope. The soil samples were chemically dried, then impregnated with polyester resin to form solid blocks. Each block was then cut and mounted, followed by grinding and diamond polishing to produce a section of uniform $30 \mu \mathrm{m}$ thickness. A typical scientific study of such soils would optically examine and describe the sections using an international system of terminology [8], allowing archaeological interpretation of the materials [9]. To develop the Ground-breaking installation, we took a more expansive direction, allowing broader cross-disciplinary interpretation. First, by examining large areas of each sample, we were able to undertake a virtual exploration of the microscale features. Second, through quantitative examination of the materials, we identified objects that provide discrete cultural signals; these objects could then be classified and spatially related. This, in turn, allowed us to develop a visual and sonic narrative that draws upon both the measurements and their interpretation as cultural signifiers.

We observed thin-section samples with a microscope at 80 -fold magnification and captured the images using a sequence of different illumination methods (Fig. 3). Each of the optical techniques produces a different resultant image (Color Plate B No. 1). The oblique incident reflected light image typically shows dark images with frequent objects that can be interpreted as related to cultural activities: specifically, burnt materials such as pieces of fired clay and partially combusted fuel materials show clearly as red and orange colors. The plane-polarized transmitted light images typically have muted colorations and may frequently show quartz sand grains as an irregular bleached-white "skin" around a near-transparent core. The abrading of these grains through exposure to the natural forces of wind and rain can result in rounded yet irregular shapes. This provides a visual clue to the relative severity of several confounded forces in

Fig. 2. Schema showing the progression from sampling cultural soil materials as intact blocks, through soil thin-section preparation, to the examination and microscope imaging of the thin section. (@ Paul Adderley and Michael Young)

Excavation and Sampling

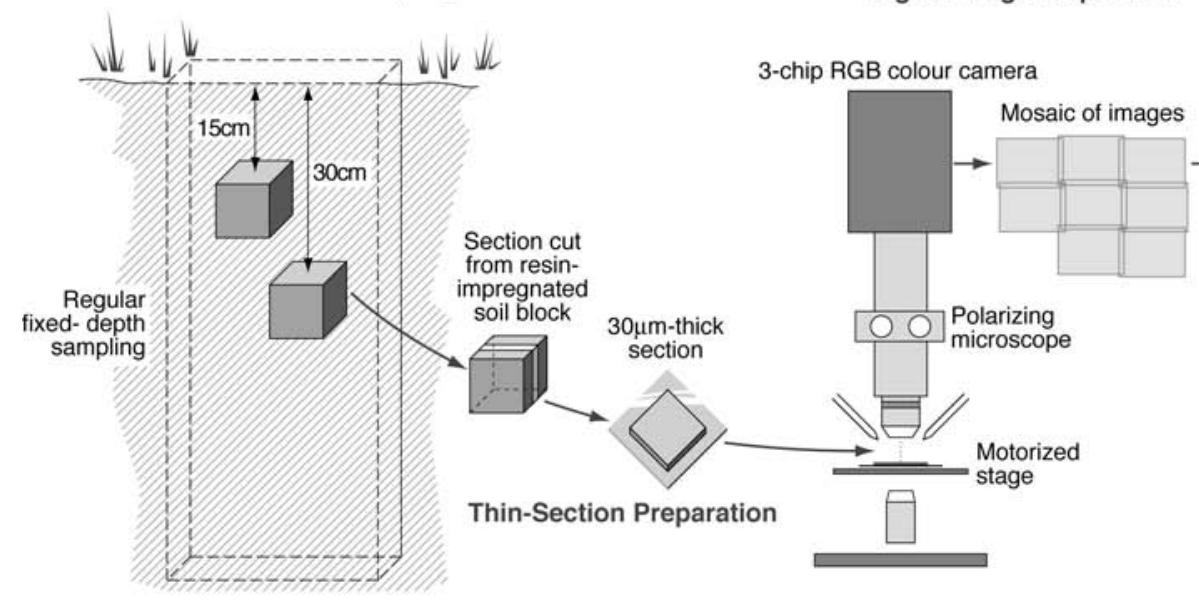

Digital Image Acquisition

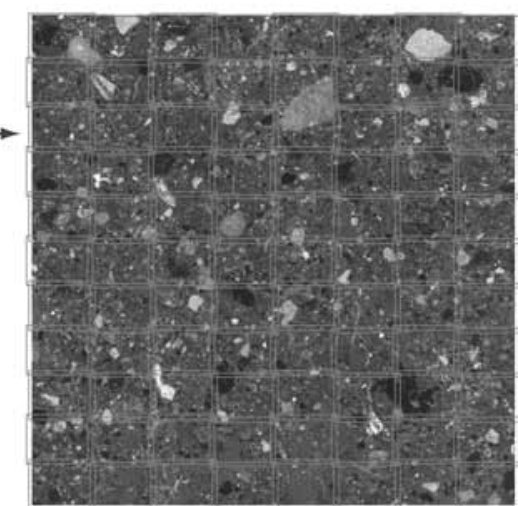

Digital image of a statistically representative area of soil thin-section 
a)

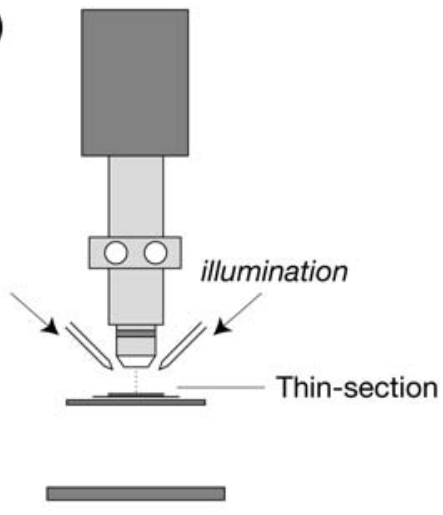

c)

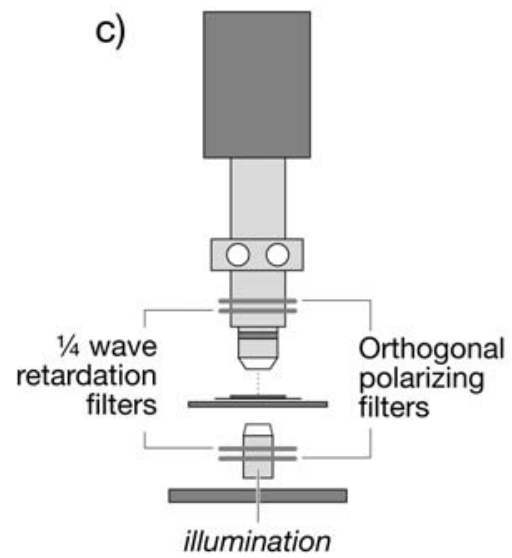

b)

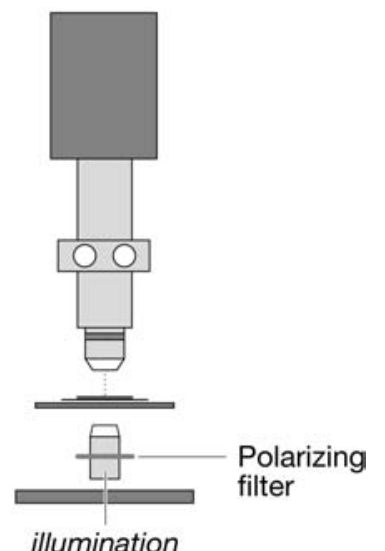

d)

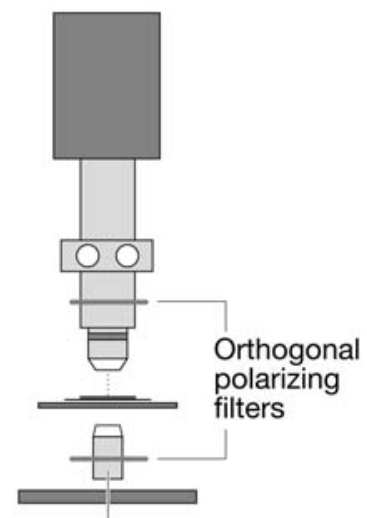

illumination

Fig. 3. Schema of the different optical methods used: (a) oblique incident illumination reflected from the sample; (b) plane-polarized illumination transmitted through the sample; (c) transmitted circularly polarized illumination; (d) with the sample between crossed polarizers. (@) Paul Adderley and Michael Young)

the natural environment. The images taken using circular-polarized illumination extend this and may show a distinct light-blue coloration in areas of the microscope slide where there is no organic or mineral material. It can, therefore, give a clear indication of the pore-spaces wherein many environmental and biological processes occur within the soil. With the sample between crossed-polarizers, all empty space in the sample will be shown black, while many mineral materials will be shown with a range of vivid birefringence colors [10]. The contrast between this image and the others frequently can reveal textural changes in the soil matrix, which may be related to human disturbance of the soil.

First we analyzed 32 areas from the eight microscope slides, each representing a discrete period in the history of the Tiwa village site. Then a large-scale calibrated image [11] was made for each sample area by capturing a mosaic of smaller images using a high-resolution Peltier-cooled digital microscope cam-

era (Fig. 2). This was repeated for all four illumination methods, for a total of 128 images. These images were then examined using image-analysis techniques [12]. The images were segmented to identify component objects of interpretative value, and measurements of these objects were taken, with each group of objects representing the imprint of a different cultural event-construction and destruction of buildings or soil disturbances such as cultivation and periods of flooding. Taking burnt materials as an example, under oblique incident illumination these materials have distinct color properties allowing a color-based segmentation of the image. Using image analysis, we identified each object in the image and analyzed its size, shape and location (Fig. 4). Thousands of objects in each image may be classified in this way. With each object defined as a collection of contiguous pixels that satisfy the segmentation criteria of specific color properties, the object's area is given by counting the number of pixels, while the outer pixels define the object perimeter. Holes within objects are identified as pixels outside the segmentation criteria. Shape is estimated by measuring parallel tangents-Feret measurements-at 360 positions around each object [13]. The fractal dimension of each object, a derived measure of shape, is calculated from the area and perimeter relationship [14]. By considering the center of the object as the point of maximum distance from any point on the perimeter, a set of coordinates for each object within an image can be determined. A data set for every identified object therefore comprises $(x, y)$ spatial coordinates and descriptors: object area, perimeter, count of the holes in the object, Feret mean, fractal dimension and color hue, saturation and intensity. These and the 10,000-year temporal dataset form the precursors for the implementation and structure of the installation.

\section{SONIFICATION AND REPRESENTATION}

Ground-breaking is a real-time, generative audiovisual work [15]. In the installation we experimented with strategies for producing visual and sonic material from complex data, as offered by this scientific study. Generating sound from scientific data or non-musical sources is a wellestablished and diverse field [16]: An aim of sonification is to render complex and multidimensional data susceptible to intuitive or more methodical appraisal and analysis, potentially via an interactive interface. What is sought, in general, is "the ability of the auditory system to extract underlying structure and temporal aspects of complex signals that are often important in scientific exploration" [17]. Our perceptual and cognitive experience of sound certainly differs radically from our response to visual stimulus, not least due to its intrinsic temporality; so, a sonic representation may foster alternative insights, so to speak, to those afforded by visual analyses. As noted by Hermann [18], the listener, in comparison to the viewer, is potentially more immediately cognizant of both global attributes and local nuances of data, even when there are simultaneous streams of information. There are examples in medicine and scientific research, such as astronomy, where otherwise hidden structural elements (e.g. wave functions) were discovered through aural analysis [19]. For this application, precision, consistency and reproducibility are essential: Sonification provides an objective, analytical experience for the listener, who may require 

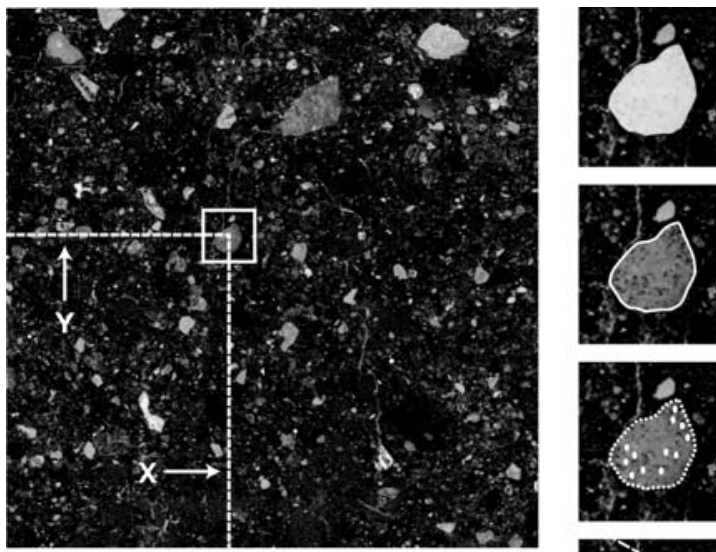

Object area

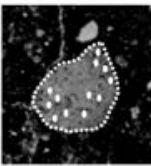

Holes within object

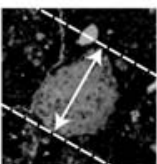

Feret (maximum)

shape measurement of object

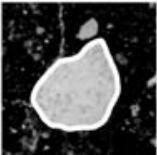

Derived fractal measurement $\left[\log _{e}\right.$ area $/ \log _{e}$ perimeter]

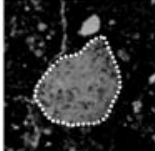

Colour measurement of object (hue, saturation, intensity)

Fig. 4. Schema of the image analysis measurements for an object segmented from an image of a soil thin section. (@ Paul Adderley and Michael Young) Each object identified by threshold pixel parameters (i.e. color definition) is then measured, with the spatial coordinates within the image, object size, color and shape parameters all reported. These data inform the sound synthesis.

appropriate training and experience to comprehend and make effective use of any given system. The translation of data to sound presents a mapping problem that explains this need for training Pre-existing wave functions are easily susceptible to sonification because they can be directly mapped with minimal intervention, such as amplification, frequency shifting or changes to playback speed. The audible result retains or mimics properties of the original (e.g in seismology) or provides a sonic metaphor (e.g. the Geiger counter). More sophisticated techniques such as physical modeling [20] retain a transpositional relationship between source data and sonic outcome.

In circumstances in which initial data are not clearly temporally or aurally orientated, "higher-level" mappings are necessary but problematic. If input data need to be radically reformulated to be congruent with a specific sound-synthesis method [21], sonification can stil be potentially accurate and consistent although inevitably the result relies substantially on the designer's intervention, whether intentional or not. The reformulation (mapping) of parameters might be quite intuitive and freely associative, even though common methods such as the direct translation, convergence or divergence of parameter sets have been noted [22]. Examples include aligning sonic events with physical gestures, common in interactive and performance media, or, in audiovisual scenarios, timbres with colors. Such mappings might be evaluated for their familiarity, effectiveness or accuracy, but only in the context of a known creative intent or after systematic learning. For sonification in general, data are not necessarily obtained from a musical/ human agent or a familiar visual context, so there may not be a commonsense or intuitive association between input and output. Any strategy for data-to-sound mapping is, therefore, as arbitrary as it is decisive.

Mapping problems are exacerbated when there is no time dimension to the source data, a situation frequently encountered in image-to-sound translation. In becoming sound, such data must be "linearized" as a temporal sequence of discrete events. Creative decisionmaking becomes paramount, entirely predicated on a desired or presumed outcome. This process is the antithesis of scientific investigation and may reveal ideologically transparent preconceptions about music, that is, what constitutes legitimate musical material and structure.
The most popular assumption is that music consists of "notes," defined by pitch and rhythm and grouped as phrases (melodies) and/or simultaneities (harmonies). Timbre is decorative rather than functional. The musical instrument digital interface (MIDI) protocols have helped formalize and ossify this reductionist view, even though in many areas of sound art practice these assumptions are rejected [23]. While MIDI offers an "offthe-shelf" approach to generative music and the exigencies of data mapping and sonification, a problem arises because the assumptions themselves-manifesting as harmonies, musical scales, rhythmic patterns, etc.-connote their own structural relations, which have a long history of cultural development and are firmly embedded in our contemporary cultural experience: These relations are far more telling than any novel patterning that might emerge from source data, which are effectively rendered redundant. This is also arguably the case in domains where other artistic assumptions are evidenced, as in electroacoustic music, electronica and post-digital and noise music. The sonified result, confusingly, might purport to offer both a neutral "scientific" re-presentation and interrogation of source data and also an artistic, positional statement inviting aesthetic response, even though the true relationship between data and sound is extrinsic to the data set itself. This object-subject confusion seems to be at the heart of sonification, in which the medium, sound, conflates the objectification of data for structural illumination with subjective and/or historically embedded "artistic" value systems.

\section{THE GROUND-BREAKING INSTALLATION}

Ground-breaking investigates these problems directly by exploring data that are essentially atemporal and visually oriented and do not in themselves invite any commonsense or culturally defined association with sound. These extracted soil data, representing a period of over 10,000 years, are complex, multidimensional and opaque. Nevertheless, these samples have a clear functional relationship to the wider historical narrative about environmental change. The installation uses both sonic and visual materials drawn from sources relevant to the Lake Chad region, presented in real time as either simple representational states or remote, modified and abstract states. The generative system driving the installation deploys these materials as 
means of interpretive sonification, in which structural relationships embedded in the data set are evidenced in these audiovisual modifications at a number of levels. The result is not a proxy for the actual data set nor intended to be related either mimetically or even metaphorically to it. Nor is there any structural musical logic applied from without. Rather, a new narrative emerges that references data both directly and obliquely, exploring a data space in which a sequence of actions can be deduced from functional descriptions of physical objects identified in the soil. This narrative develops structural and syntactical relationships that are consistent and, in theory, comprehensible. It is intended to engage the audience without the need for prior training or direct experience; listening and understanding are thereby considered different to rational aural analyses.

The installation encapsulates ideas of open-endedness and periodicity, bringing together indeterminate and cyclical processes. The macrostructure of the installation (Fig. 5) is guided by a master clock, which represents the entire period of 10,000 years. Our chief aim with the installation is to provide an interpretation of detailed soil data files that temporally correlate to this period. The equivalent time interval executed by the installation system varies on each cycle, resulting in presentation of the 10,000 years in periods of between 20 and 50 minutes. Each cycle is denoted by data, interpolated where necessary, that describe the level of Lake Chad at decadal intervals. The levels are indicative of three states: flood, drought or human population. These data are interpreted as a probability function that influences the generative processes of the installation, by adjusting the likelihood or specific character of the various visual and sonic events that are in evidence over each cycle of the system (see the Stochastic Processor in Fig. 5). This stochastic approach ensures that each cycle of the installation is unique and will only deploy a selection of materials and processes available to the system as a whole. Adaptive probability systems have been applied in other recent work as a means of refining and structuring generative behaviors [24]

The visual component comprises two elements, a library of on-site photographs and the thin-section images of soil samples. At irregular time intervals-determined by a secondary clock-a thin section is selected and its four associated micrograph images and soil data files are loaded into the system. The choice of thin sections is random- ized but restricted to those relevant to the time period indicated by the current position of the master clock. The images are loaded in fours to explore the contrasting and complementary perspectives offered by the different optical imaging methods. An automated, fourdimensional cross-fade between images runs continuously, creating a rhythmic rebalancing of the color and detail intrinsic to the images. The installation is also able to zoom closely onto individual soil fragments that are identified by $(x, y)$ coordinates and described in each data file and then to scan between data points for real-time animation. These and other visual devices, including presentation and manipulation of on-site images, are also influenced by the probability functions that reflect the currently selected lake-level state: drought (static displays, coloration effects), flood (crossfading of soil images and flowing progression between data points) and human popula- tion settlement (dynamic zoom activity, on-site photographic material).

The choice and manipulation of sound materials are similarly influenced. There are clearly referential sounds, taken from the Lake Chad region: a range of environmental recordings; human voices in conversation, at work and home; as well as recordings of live music. These materials are much more likely to be invoked by the system when they are relevant to the historical/temporal scale, unfolded by the master clock. Audio samples obtained from alternative "non-human" sources, such as flowing water, and tactile interactions with gravel particles, etc. are suggestive only of a generalized idea of materiality or energy; these are also deployed when appropriate. As with the images, the specific choices and timings are variable and differ on each cycle.

The data files identify and characterize discrete objects in the soil: data that suggest the presence of certain materi-

Fig. 5. Schema of the macrostructure of the Ground-breaking installation. (ㄷ Paul Adderley and Paul Young) Note the close integration of the different data sets: the thin-section images of the cultural soils both form a visual element and provide the source image-analysis data subsequently used in the sound synthesis.

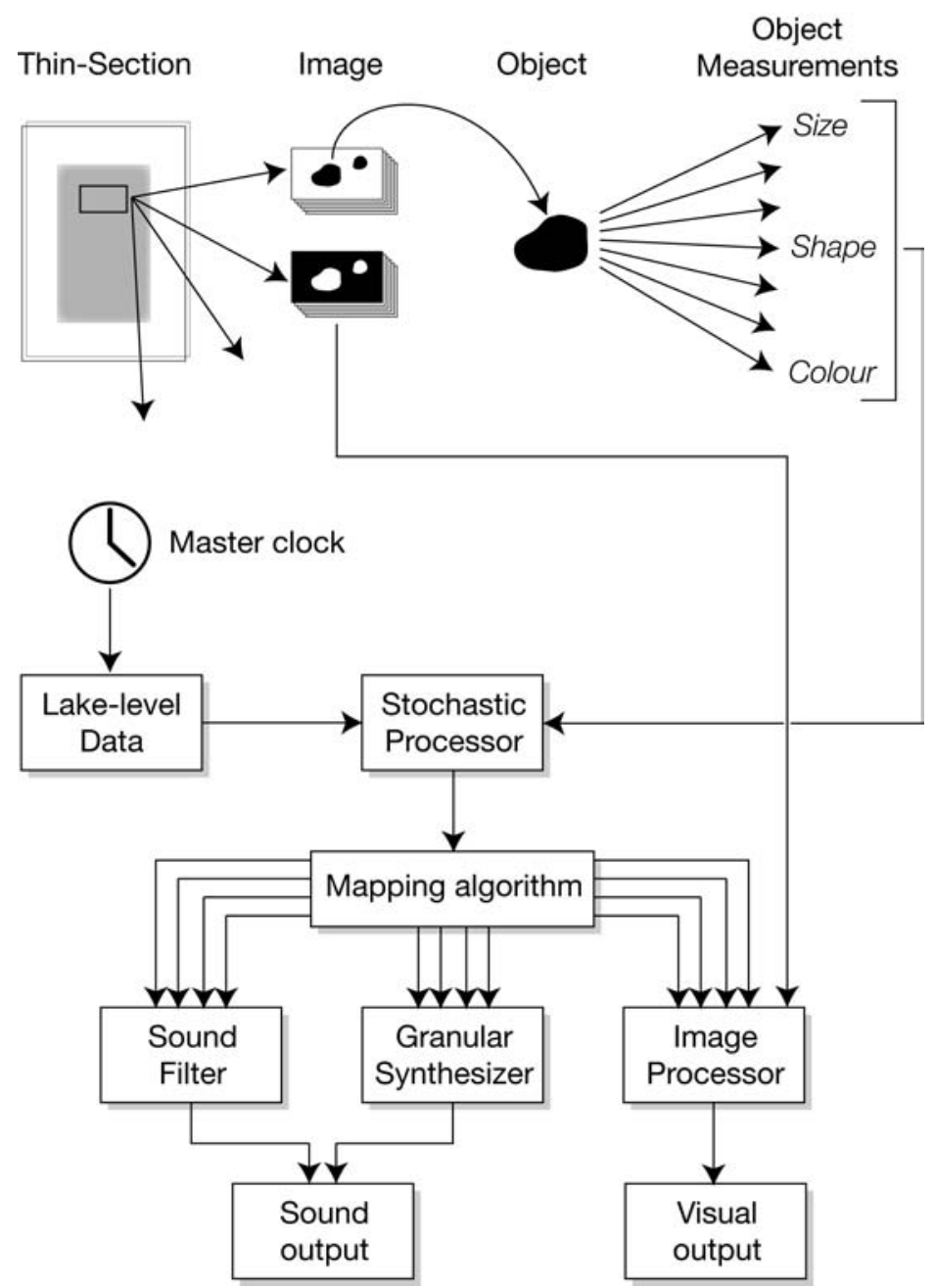


als in the environment, developing our knowledge of the period in question. Each soil thin section may contain several thousand objects; the data files are large, comprising an eight-dimensional array of some 2,000-6,000 lines. Thus there are two challenges: the sheer profundity of data and their visual terms of reference, which fail to suggest any particular context for sonic representation. In Ground-breaking, the mapping is arbitrarily assigned but it is consistent in any one cycle of the 10,000-year history. For each iteration of the master-clock cycle, the mapping is reshuffled and assigned autonomously by the system with no further user intervention (see the mapping algorithm in Fig. 5). One-toone, convergent and divergent mapping strategies are used; the strategy and actual assignment map are generated and regenerated randomly. As there are eight dimensions and over 20 sound-synthesis parameters, this reshuffling is decisive in predetermining the vocabulary and behavior of sounds and ensures that a new interpretative perspective is offered in each cycle. Over time, the entire body of data will be visited and exploited.

Any given set of soil data is explored for synthesis as long as the current image selection is in view; this time-based, iterative behavior is itself drawn from one of the object descriptors. Two synthesis techniques are employed: subtractive synthesis (multi-band filtered noise) and granular synthesis (or granular reconstruction, the production of extensive and timbrally rich sound events and textures by the proliferation of tiny sonic fragments or grains). Granular methods offer the possibility of both clear sonic references and more abstract, acousmatic [25] material, depending on the content, duration and processing of individual grains. The filtering techniques can be employed on both a noise signal and the sound product of the granular synthesis. Sonic parameters include the frequency content, bandwidth and har monicity of filter banks, the size, content, amplitude and post-processing of grains. Grain content is particularly critical: This is obtained from an existing library of recorded sounds stored by the system. The library sounds are tagged with descriptors denoting their referential content (e.g. water, work, environment, human voice, music) and their sonic characteristics (e.g. loudness, brightness, roughness, pitch-noise). The tags are assigned as sound parameters, so that appropriate sound sources are looked up and read into the granular synthesizer as a basis for subsequent events and textures. The result draws the listener into imaginative engagement with geographical/cultural references and abstract processes of change. The narrative constructed in Ground-breaking is an interpretative sonification, the result of a stochastic exploration of the soil data sets as "cultural indices” (returning to Barthes's terminology).

\section{CONGLUSION}

Commonly held present-day uncertainties about suitable societal responses to the climate-change debate underscore the need to seek new and novel conceptualizations of environmental change. The Ground-breaking installation was commissioned to try to address this; through it we seek to raise awareness of the scientific aspects of how people have coped, regardless of causation, with environmental change in the African Sahel. In so doing, we acknowledge the problems and potential contradictions in data representation.

By inviting the audience to develop their own understanding of a narrative, the work has promoted a critical appraisal of the data sets used, their representations and any prior perceptions of the African Sahel. As such, direct deduction of the human processes or environmental events is not explicitly sought in Ground-breaking; the installation remains an unresolved generative work. In this form, it propels the audience toward a liminal space, an understanding of place and of rates of change. It has attempted to breach the barriers presented by different temporal and spatial scales: between landscape and the production of artifacts, between the scientific analysi of artifacts and their manufacture, and between micro-scale information and macro-scale evidence of the extremes of climatic change.

The narrative developed in Ground breaking explores a data space that contains a set of deduced actions from functional descriptions of physical objects; this has revealed the possibility that a yet more detailed data analysis and deeper data space exploration can produce further novel insights into the nature of the cultural imprints held by the soil. The Ground-breaking installation also highlights the transferability of the meta-constructs of the narrative to other domains, including other geographical environments and their cultural imprints.

\section{Acknowledgment}

The Ground-breaking installation was commissioned from the authors and supported by Research Councils UK. The authors gratefully acknowledge the use of ethnographic recordings by Holger Kirscht, University of Cologne. From the University of Stirling, Bill Jamieson kindly provided technical assistance.

\section{References and Notes}

Unedited references as provided by authors.

1. Ground-breaking was first shown at National Museum of Scotland, Edinburgh, 9-16 March 2007, with support from the Research Councils UK.

2. Material about the installation, photographs and video extracts are available at $<w w w . g r o u n d-$ breaking.net $>$.

3. The Sahel region is commonly considered the belt of land fringing the southern extreme of the Sahara desert extending from the Atlantic coast in Senegal desert extending from the
through to eastern Chad.

4. R. Barthes (1977) Image, Music, Text. London: Fontana Press.

5. W.P. Adderley et al. (2004) Enhancing ethnopedology: integrated approaches to Kanuri and Shuwa Arab definitions in the Kala-Balge region, Shuwa Arab definitions in the Kala-Ba

6. C. Magnavita (2004) Zilum-Towards the emergence of sociopolitical complexity in the Lake Chad region (1800 BC-1600 AD). In M. Krings and E. Platte (Eds.). Living with the Lake. Perspectives on History, Culture and Economy of Lake Chad. Cologne: Rüdiger Köppe Verlag, 73-100.

7. G. Connah (1981) Three Thousand Years in Africa: Man and His Environment in the Lake Chad Region of Nigeria. New York: Cambridge University Press, pp. $27-76$.

8. P. Bullock et al. (1985) Handbook for Soil Thin Section Description. Wolverhampton: Waine Research Publications.

9. M-A. Courty et al. (1989) Soils and Micromorphotogy in Archaeology. Cambridge: Cambridge University Press, pp. 104-137

10. Birefringence is the name given to the optical phenomenon of double refraction due to differen directional light transmission properties of the material examined. In this instance, crystalline materia in the soil may exhibit a range of colors.

11. W.P. Adderley et al. (2002) Color description and quantification in mosaic images of soil thin sections. Geoderma, 108: 181-195.

12. For theory, see J.C. Russ (2006) The Image Processing Handbook (Fifth Edition) CRC Press, Boca Raton, Florida. For implementation: Adderley et al. [11].

13. The Feret statistic is defined as the measured distance between theoretical parallel lines drawn tangent to the edge of the individual soil particle of interest. See C.P. Murphy et al. (1977) The measurement and characterization of voids in soil thin sections by image analysis. Part I. Principles and techniques. Journal of Soil Science, 28, 498-508, and Russ [12].

14. B.B. Mandelbrot (1983) The Fractal Geometry of Nature. San Francisco: W.H. Freeman, pp. 109-130.

15. The work was implemented in Max/MSP and Jitter software (Cycling '74 [2006] MAX Reference Manual 4.6/7. San Francisco: Cycling '74 Inc. <www. cycling74.com>)

16. C. Scaletti (1994). Sound synthesis algorithms for auditory data representations. In G. Kramer (Ed.) Auditory Display: Sonification, Audification, and Audi tory Interfaces. Addison-Wesley. 223-251. 
17. R. Bargar et al. (1999) Sonification Report: Status of the Field and Research Agenda. International Community for Auditory Display National Science Foundation Report. See: <dev.icad.org/node/400>.

18. T. Hermann and H. Ritter (1999) Listen to your Data: Model-Based Sonification for Data Analysis. In S.M. Rahman, et al. (Eds.) Advances in Intelligen Computing and Multimedia Systems. Baden-Baden: International Institute for Advanced Studies in System Research and Cybernetics, pp. 189-194

19. Bargar et al. [17]

20. Hermann and Ritter [18].

21. Hermann and Ritter [18].

22. A. Hunt and M.M. Wanderley (2002). Mapping performance parameters to synthesis engines. Organized Sound, 7:2, 103-114.
23. See for example past discussions by R. Moore 1988) The Dysfunctions of MIDI. Computer Musi Journal, 12:1, 19-28. and Wishart, T. (1996) On Sonic Art 2nd Ed. Amsterdam: Harwood Academic.

24. M. Young (2008) NN Music: Improvising with a 'Living' Computer. In R. Kronland-Martinet et al (Eds.) Computer Music Modeling and Retrieval: Sense of Sounds. Lecture Notes in Computer Science 4969. Berlin: Springer-Verlag, pp. 337-350.

25. Wishart [23]

Paul Adderley is an RCUK Academic Fellow engaged in environmental and geoarchaeological research. He studies long-term soci etal-climatic interactions, the sustainability of past societies and environmental risk using novel methodologies. He was a National Endowment for Science, Technology and the Arts (NESTA) awardee in 2004. His recent work has included examining fresh ways of considering the outputs of scientific study.

Michael Young is a senior lecturer and com poser with interests in computer music, in teractive performance systems, improvisation and machine learning. He is co-director of the Live Algorithms for Music network. Recent compositions include Argrophylax (2005), Aur(or)a (2006), Piano Prosthesis (2007) and Oboe_Prosthesis (2008). See: <www. michaelyoung.info>.

\section{Smart Textiles: Science and Technology of Textile Art}

Leonardo is pleased to announce a new special project in the topic area of Smart Textiles. This project expands upon Leonardo's archive of textile art documentation by focusing on textile artists and scientists around the world who work with smart textiles or the new textiles science and technology.

Artists and researchers interested in writing about their work involving the science and technology of smart textiles and clothing arts are invited to view the Leonardo Editorial Guidelines and related information at $<$ leonardo.info/Authors> and send in a manuscript proposal to <leonardomanuscripts@gmail.com>.

To view a list of papers published in Leonardo and Leonardo Music Journal on topics related to textile arts, please see: <leonardo.info/isast/journal/calls/smartextiles_call.html>.

This project is supported by the Marjorie Duckworth Malina Fund, which honors the memory of a key longtime supporter of Leonardo/ISAST. The project recognizes Marjorie's dedication to the ideals of international cooperation by emphasizing the participation of artists throughout the world. For information on making a donation to Leonardo/ISAST in memory of Marjorie Duckworth Malina, please visit <http://leonardo.info/isast/donations.html>. 